\title{
Diagnosis of COVID-19: Considerations, controversies and challenges
}

\author{
K Dheda, ${ }^{1,2}$ MB BCh, FCP (SA), FRCP (UK), PhD; S Jaumdally, ${ }^{1} \mathrm{MPH}, \mathrm{PhD}$; M Davids, ${ }^{1}$ BSc (Hons), PhD; J-W Chang, ${ }^{1}$ BSc (Hons), MSc, PhD; \\ P Gina, ${ }^{1}$ MB BCh, FCP (SA); A Pooran, ${ }^{1}$ BSc (Hons), MSc, PhD; E Makambwa, ${ }^{1}$ MB BCh, FCP (SA); A Esmail, ${ }^{1}$ MD, FCP (SA); \\ E Vardas, ${ }^{3} \mathrm{BSc}$ (Hons), MB BCh, DTM\&H, DPH, MMed (Virology), FC Path Clinical Virology; \\ W Preiser, ${ }^{4,5}$ Dr Med, Dr Med Habil,WP, DTM\&H, MRCPath \\ ${ }^{1}$ Centre for Lung Infection and Immunity, Division of Pulmonology, Department of Medicine and UCT Lung Institute and South African MRC/UCT Centre for \\ the Study of Antimicrobial Resistance, University of Cape Town, South Africa \\ ${ }^{2}$ Faculty of Infectious and Tropical Diseases, Department of Immunology and Infection, London School of Hygiene and Tropical Medicine, UK \\ ${ }^{3}$ Lancet Laboratories, Johannesburg, South Africa \\ ${ }^{4}$ Division of Medical Virology, Department Pathology, Faculty of Medicine and Health Sciences, Stellenbosch University, Cape Town, South Africa \\ ${ }^{5}$ National Health Laboratory Service, Cape Town, South Africa
}

Corresponding author: K Dheda (keertan.dheda@uct.ac.za)

\begin{abstract}
Coronavirus disease 2019 (COVID-19) due to a novel virus, severe acute respiratory syndrome coronavirus 2 (SARS-CoV-2), is a global pandemic that has resulted in over 1.5 million confirmed cases and close to 100000 deaths. In the majority of symptomatic cases, COVID-19 results in a mild disease predominantly characterised by upper respiratory tract symptoms. Reverse transcription polymerase chain reaction (RT-PCR) using a nasopharyngeal sample is the mainstay of diagnosis, but there is an $\sim 30 \%$ false negative rate early in the disease and in patients with mild disease, and therefore repeat testing may be required. RT-PCR positivity can persist for several days after resolution of symptoms. IgM and IgG antibody responses become positive several days after the onset of symptoms, and robust antibody responses are detectable in the second week of illness. Antibody-based immunoassays have a limited role in the diagnosis of early symptomatic disease. However, their incremental benefit over RT-PCR in the first 2 weeks of illness is currently being clarified in ongoing studies. Such assays may be useful for surveillance purposes. However, their role in potentially selecting individuals who may benefit from vaccination, or as a biomarker identifying persons who could be redeployed into essential employment roles, is being investigated. Rapid antibody-based immunoassays that detect viral antigen in nasopharyngeal samples are being developed and evaluated.
\end{abstract}

Afr J Thoracic Crit Care Med 2020;26(2):Published online 21 April 2020. https://doi.org/10.7196/AJTCCM.2020.v26i2.099

Coronavirus disease 2019 (COVID-19) due to a novel virus, severe acute respiratory syndrome coronavirus 2 (SARS-CoV-2), is now a global pandemic. There are more than 1.5 million confirmed cases across almost every country in the world, and at the time of writing (early April 2020) there were close to 100000 recorded deaths. ${ }^{[1]}$ The diagnosis of COVID-19 can be challenging, and as with any disease entity, a number of factors, including disease stage, disease prevalence, patient profile and sample type and quality, can influence diagnostic test performance. In this review we outline the performance outcomes of key tests used to diagnose COVID-19, and considerations that modulate performance. The safety of healthcare workers collecting samples, laboratory safety aspects and experimental approaches such as detection of volatile organic compounds in exhaled breath, mass spectrometry studies of different sample types, methods of signal amplification and utility of other novel approaches are not discussed here.

\section{Indications for testing}

Country-specific indications and criteria for testing have evolved rapidly, and are being updated as information emerges and as the epidemic progresses. These recommendations have been guided by the phase of the epidemic and available resources. Generally speaking, testing for COVID-19 should currently be considered in anyone with symptoms of an acute respiratory tract infection (upper or lower) and with or without systemic symptoms such as fever, fatigue and myalgia. ${ }^{[2-5]}$ In mild disease, testing directs the need for self-isolation and identification of new cases through contact tracing and testing of contacts. As the epidemic progresses, and with forecasted limited testing capacity, testing may be directed to specific subgroups or those with enhanced risk of a poor outcome.

\section{The differential diagnosis}

In the clinical setting, COVID-19 will form part of the differential diagnosis of any acute respiratory presentation, including infectious causes of pneumonia (e.g. bacterial, influenza, other viral pneumonia, pneumocystis pneumonia, tuberculosis (TB), etc.), acute exacerbations of asthma and chronic obstructive pulmonary disease (COPD), acute pulmonary embolism, cardiac failure and other conditions. Relevant investigations will depend on the clinical context, and will likely include pulmonary imaging, relevant laboratory investigations, blood cultures and interrogation of urine and/or lower respiratory tract specimens to rule in a viral, mycobacterial, fungal and/or bacterial cause. Clinical 
and laboratory parameters that may suggest viral infection include pyrexia, acute malaise and myalgia, and lymphopenia. C-reactive protein is unhelpful in distinguishing COVID-19 from other infections. Procalcitonin is elevated in severe COVID-19 and when there is secondary bacterial infection. ${ }^{[6,7]}$ In early disease low procalcitonin may distinguish COVID-19 from bacterial infections, but not from other viral diseases (data are awaited to confirm this supposition). In those with underlying asthma or COPD the presence of pulmonary infiltrates may favour a respiratory infection-related cause, though a cardiac cause must also be considered in the differential diagnosis.

\section{The biological sample of interest}

The most common sample types sent for testing, usually by means of reverse transcription polymerase chain reaction (RT-PCR), are nasopharyngeal and oropharyngeal samples obtained using a swab, placed in viral transport medium. There is already considerable shortage of reagents (and swabs), meaning that dry swabs are being sent to laboratories in some centres. Dry swabs are less costly and more conducive to community-based testing, but data are urgently required to determine the comparative sensitivity of dry swabs compared with using viral transport medium (taking into account the time from sample acquisition to sample processing). Samples from the lower respiratory tract including sputum, tracheal aspirate, bronchial washings and bronchoalveolar lavage may also be sent. In patients with COVID-19 disease, samples from the lower respiratory tract are more likely to test positive (discussed below). Viral RNA can also be detected in stool in $\sim 30 \%$ of cases, and in blood in $\sim 1 \%$ of cases, ${ }^{[8]}$ but rarely in urine.

\section{Clinical and immunodiagnostic trajectory of COVID-19 and sampling considerations}

Recent data from infections in special contexts such as cruise liners ${ }^{[9]}$ and in close contacts of COVID-19 patients ${ }^{[10]}$ have demonstrated that SARS-CoV-2-specific RT-PCR may be positive in the early phase of the disease, and that viral shedding in the asymptomatic phase and in the early prodromal phase can be considerable. ${ }^{[11,12]}$ At present, screening of asymptomatic individuals by RT-PCR has been constrained by limited testing capacity, and the need to focus public health efforts and resources on symptomatic persons.

In symptomatic individuals, $80-90 \%$ of patients have mild symptoms not requiring hospitalisation. Depending on age and the presence of risk factors, $\sim 10-20 \%$ of symptomatic persons may require admission to hospital because of respiratory or other complications. Individuals in this enhanced risk category may have one or more such factors, including age $>50$ years, comorbidities, history of significant tobacco smoking and underlying immunocompromising illnesses. ${ }^{[10,13]}$ In mild disease, especially in the early stages, the RT-PCR false negative rate is $\sim 30-40 \%{ }^{[8,14,15]}$ A meta-analysis reported that a single test $\sim 10$ days post symptom development had an $\sim 33 \%$ false negative rate using a nasopharyngeal swab (52.89\% for a throat swab). ${ }^{[16]}$ Ai et al. ${ }^{[17]}$ reported a false negative rate of $41 \%$ in a cohort of 1014 hospitalised patients; the estimated median (standard deviation) interval between the initial negative test and subsequent positive RT-PCR result was 5.1 (1.5) days. A selection of other studies reported false negative rates of between 3 and 29\%. ${ }^{[18-20]}$ Notably, some patients required up to five repeat tests before a positive result was ascertained. ${ }^{[19]}$ This false negativity phenomenon may be the result of several factors, including a low viral load below the detection limit of the assay, low sample volume or cellular mass during acquisition, sampling location (upper v. lower respiratory tract), sample degradation during transport or storage, sample processing methodology and the timing of sampling in relation to the stage of the disease (RT-PCR positivity may progressively increase during the course of the disease) ${ }^{[14]}$

Test accuracy will depend on the quality of the specimen collected. ${ }^{[20]}$ It has since been shown that specimens from the lower respiratory tract have a higher viral load, and are hence more likely to test positive than specimens from the upper respiratory tract. ${ }^{[8,21]}$ Nasopharyngeal specimens have better yield than oropharyngeal samples. ${ }^{[8,15]}$ In hospitalised patients with severe disease, Wang et al.$^{[8]}$ found a sensitivity of $93 \%$ in bronchoalveolar lavage fluid (a high aerosol-generating procedure), $72 \%$ in sputum and $63 \%$ in nasal swabs; sensitivity ranged from 0 to $32 \%$ in pharyngeal swabs, faeces, blood and urine. Given these considerations, a negative test from an upper respiratory tract specimen should be repeated after 1 - 3 days (the optimal timing is unclear), or a lower respiratory tract specimen obtained to exclude a false negative result, if clinical suspicion is high. ${ }^{[22]}$

Viral shedding in asymptomatic, early prodromal and minimally symptomatic individuals, and after resolution of symptoms, helps to explain the rapid and extensive spread of COVID-19. In patients with more severe disease, including those with lower respiratory tract infection, but also in individuals with mild disease, high viral loads can often be detected for several days after the resolution of symptoms. ${ }^{[23]}$ The significance of this remains unclear, although recent data from a limited number of patients suggest that RT-PCR positivity does not necessarily mean shedding of infectious virus after symptom resolution. ${ }^{[1]}$ Zhifeng et al. ${ }^{[24]}$ demonstrated that RT-PCR using nasopharyngeal samples can be negative even when there is computed tomography scan evidence of COVID-19 pneumonia. ${ }^{[24]}$ Ali et al. ${ }^{[17]}$ showed an improvement in disease extent in $42 \%$ of CT scans prior to RT-PCR tests becoming negative. It is unclear whether patients whose symptoms have resolved but who continue to have detectable viral RNA in respiratory samples can transmit infection. Furthermore, when symptoms have resolved, and especially given limited testing capacity, it remains unclear when patients may be discharged from the intensive care unit into the general ward setting, or from hospital into the community, especially if there are other individuals with high-risk profiles living in the same household. Therefore there are no clearly defined guidelines as to when it is safe for social mixing to occur after symptoms have resolved. Healthcare worker safety must be taken into account when collecting sputum, which should ideally be performed in an infection-controlled environment, or in the open air in ambulant patients.

\section{RT-PCR assays and their performance}

Currently, RT-PCR is the (imperfect) 'gold standard' for the diagnosis of COVID-19. The development of molecular detection assays has been facilitated by the sequencing of SARS-CoV-2. ${ }^{[25]}$ The assay consists of two principal steps: ( $i$ ) viral RNA extraction from patient specimens performed manually or using automated platforms; and (ii) reverse transcription and PCR amplification using specific primers and specific probes for real-time detection (see Fig. 1 for an overview). 


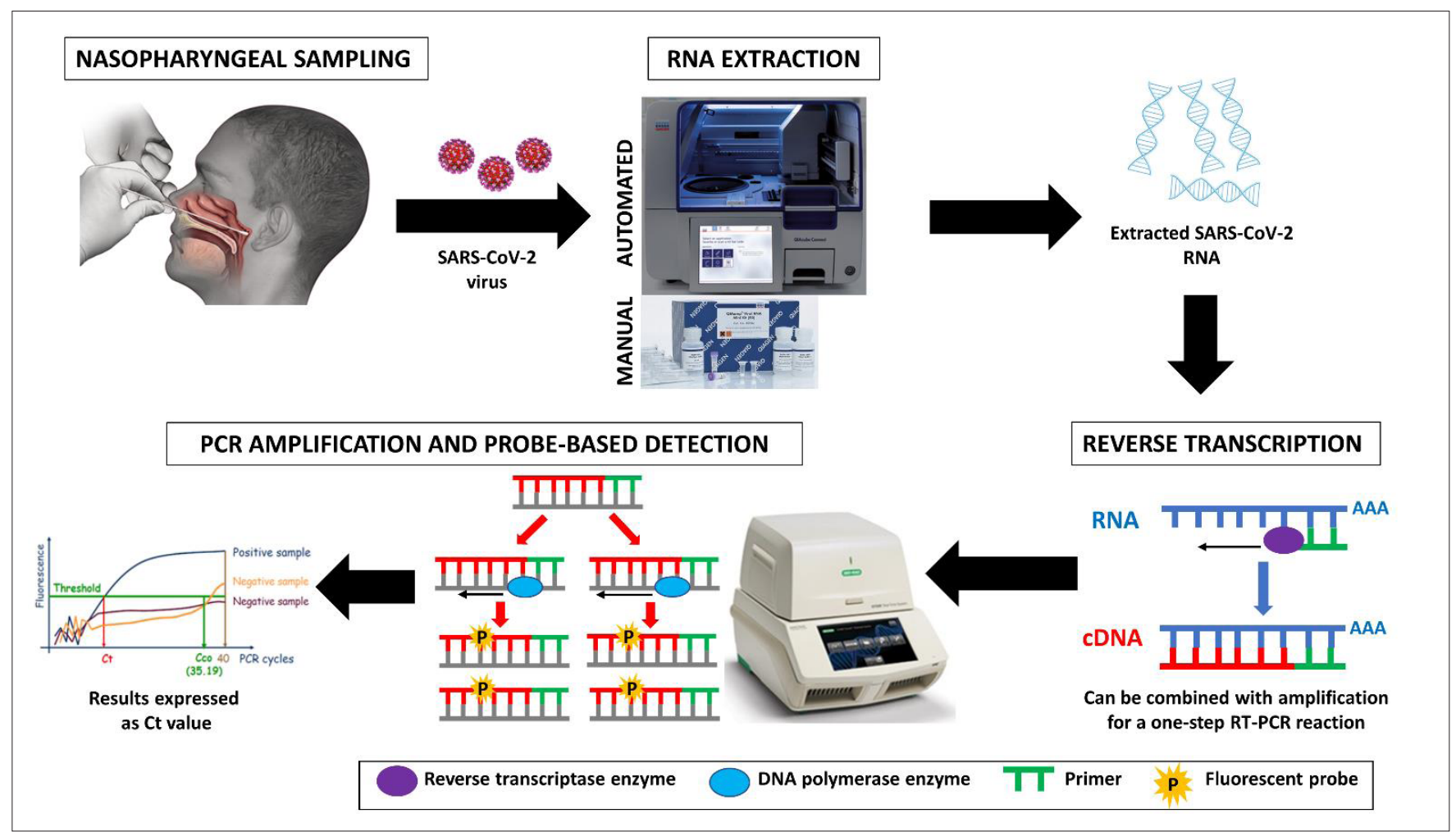

Fig. 1. Overview of severe acute respiratory syndrome coronavirus 2 (SARS-CoV-2) reverse transcription polymerase chain reaction (RT-PCR)-based detection. Viral RNA extraction is performed from nasopharyngeal/oropharyngeal specimens using a manual or automated platform. RT-PCR is performed in a two-step assay. Extracted RNA is first reverse transcribed to make complementary DNA (cDNA). The cDNA is then amplified in the second step and fluorescence results from 5'-3' exonuclease cleavage of a fluorescently-labelled target-specific probe enabling DNA amplification at each PCR cycle. $(C t=$ cycle threshold $)$.

The use of robotic systems allows for increased throughput for RNA extraction and PCR setup. Because of current resource constraints (trained personnel and reagents) and the necessity to rapidly deliver test outcomes, most diagnostic laboratories are skipping the postextraction RNA quality and quantification check, which is costly and labour intensive.

Several SARS-CoV-2 targets are being used, and these include the envelope, nucleocapsid and RNA-dependent RNA polymerase (RdRp) genes, and two large open reading frames orfla/orflb, and Ribonuclease $\mathrm{P}^{[4]}$ Generally, at least two target genes need to be identified for SARS-CoV-2 confirmation. However, interpretation algorithms differ with respect to the number of genes that need to be detected for the test to be considered positive. For some protocols, results are interpreted as indeterminate or negative if one of the genes is not detected, whereas for others, identification of one gene is used as screening test, while that of the subsequent gene(s) serves as a confirmatory test. ${ }^{[4]}$ From a laboratory perspective, multiplexing of targets allows for better efficiency, shorter turnaround times and more optimal management of laboratory consumables. ${ }^{[26]}$ Vogels et al..$^{[27]}$ evaluated nine primer-probe sets. They confirmed that each pair had a detection efficiency of $>90 \%$, but there were differences in the ability to differentiate true negatives from positives in patients with a low viral load. Some sets led to inconclusive results due to nonspecific background amplification (including the initial sets issued by the US Centers for Disease Control and Prevention but with subsequent rectification). With viral evolution, nucleotide substitutions may emerge that could affect primer/probe binding regions that could alter the sensitivity of PCR. Indeed, a potentially problematic mismatch in the RdRp-SARSr reverse primer has already been confirmed. The threshold cycle value of the target gene remains the quantitative endpoint to ascertain viral load and, depending on the kit used, this value generally lies in the 30 - 40 range. ${ }^{[4,27]}$ To control for nonspecific PCR inhibition, an internal positive amplification control (e.g. SARS-CoV-2 E-gene RNA, SARSCoV Frankfurt 1 RNA) is included in the assay, while a negative control interrogates for contamination during sample preparation.

Digital PCR (dPCR) has been used to perform a quality assurance verification of RT-PCR. ${ }^{[28]} \mathrm{dPCR}$ involves partitioning a sample into many individual parallel PCR reactions, allowing even a single molecule to be amplified more than a million-fold. Using this technique, sensitivity was significantly improved from $28.2 \%$ by RTPCR to $87.4 \%$ by RT-dPCR. ${ }^{[28]}$ Moreover, $15 / 16$ close contacts in South Korea that were Guidelines for Laboratory Diagnosis of Coronavirus Disease 2019 (COVID-19)-inconclusive with conventional RT-PCR (likely because not all the targets of interest were detected) were dPCRpositive. The overall sensitivity, specificity and diagnostic accuracy of RT-dPCR was $90 \%, 100 \%$ and $93 \%$, respectively. Moreover, the higher sensitivity of RT-dPCR translated into detection of viral RNA for longer periods than with conventional RT-PCR in convalescing patients. While RT-dPCR is more sensitive and suitable for detecting low viral loads, its accessibility is limited by the complexity of the system and cost implications, and the inability to multiplex target genes of interest. ${ }^{[29]}$ 
Several automated rapid nucleic acid amplification tests have recently received Food and Drug Administration (FDA) approval for emergency use. Cepheid's Xpert Xpress SARS-CoV-2, run on the Gene Xpert platform, detects multiple gene targets and can provide a result within 45 minutes (https://www.cepheid.com/coronavirus). Abbott's rapid COVID-19 test, run on the Abbott ID NOW device, can provide results within 13 minutes (https://www.alere.com/en/home/productdetails/id-now-covid-19.html). The former may be convenient in countries such as South Africa (SA) that have an extensive Gene Xpert infrastructure, and the technology lends itself to onsite point-of-care testing using portable Xpert platforms such as Xpert Edge.

While RT-PCR currently remains the imperfect gold standard for the rapid confirmation of SARS-CoV-2 infection, ongoing genetic evolution of the virus highlights the need to closely monitor and review the methodology based on emerging data. It is possible that a better stage-specific reference standard may emerge incorporating immunoassay results.

Limited testing capacity remains a challenge to widespread surveillance and testing in SA. Expanding testing services to researchbased laboratories is fraught with challenges, including the need for accreditation of laboratories (by the South African National Accreditation System in SA) and personnel (by the Health Professions Council in SA). Capacity shortfalls are further compounded by an international and countrywide shortage of kits and reagents and a severe reduction in international freight shipping capacity. However, the implementation of rapid automated molecular testing (Xpert Xpress SARS-CoV-2) will be helpful if enough cartridges can be procured.

\section{Immunoassays and their utility}

Several antigen-based immunoassays have been developed that detect antibodies in serum or plasma. ${ }^{[30]}$ One such assay was recently FDA approved, and the Foundation for Innovative New Diagnostics website lists over 200 companies that are either making or have made such assays. ${ }^{[31]}$ Some are rapid lateral flow assay (LFA)-based while others are enzyme-linked immunosorbent assay (ELISA)-like tests. Both formats have antigen impregnated on a test line or on a plasticplate surface, and detect human IgG or IgM, and sometimes also IgA antibodies. In the meantime, rapid capture assays that detect viral antigens in nasopharyngeal aspirates have also been developed, and are being evaluated in tandem.

Despite the proliferation of different testing devices and kits that are emerging, there are very few independent validation data on which specific assays work optimally. Therefore the sensitivity, specificity and predictive values of individual tests in different contexts remain unknown. A web-based resource has been developed that lists assays that have now been approved for use in specific countries. ${ }^{[32]}$ Some tests purchased by specific countries have already been found not to meet expectations. ${ }^{[33]}$ In Spain, one of worst-hit European nations, health authorities purchased thousands of rapid serological tests from a biotech company in China, but these were later found to have a sensitivity of $\sim 30 \% .{ }^{[34]}$ SA companies have already produced iterations of LFA platforms, and they are currently being evaluated.

One concern is test specificity, as there are four common human coronaviruses that cause up to a third of common cold episodes. Poorly designed antibody tests may cross-react with pre-existing anti- coronavirus antibodies. High false-positivity rates may erroneously indicate disease in those without COVID-19, resulting in wasted public health contact-tracing efforts, unnecessary anxiety and even worse, unintended exposure of individuals to COVID-19 in testing centres and wards if they are hospitalised. Sub-optimal sensitivity with LFA formats without a signal amplification step is a potential concern as LFA, depending on the context, may have suboptimal sensitivity compared with ELISA-based assays. However, to what extent this applies to COVID-19 remains to be seen.

Several recent articles describe longitudinal antibody responses in patients with COVID-19. ${ }^{[35-38]}$ Broadly speaking, IgM responses tend to become detectable 3 - 7 days after the onset of symptoms. ${ }^{[37,39]}$ Robust responses generally develop during the second week of illness. ${ }^{[35,36]}$ Given these considerations, antibody-based tests are not recommended for first-line diagnosis within the first few days of symptoms. There is some evidence that combining antibody and RTPCR data during the early phase of disease may be useful and may have some incremental benefit, though further studies are required. However, the SA Health Products Regulatory Agency and other agencies have recently indicated, based on guidance from the SA National Institute of Communicable Diseases and the World Health Organization, that serological testing is not suitable for diagnosis of acute SARS CoV2 infection, and should be limited to epidemiological surveys (at least until more data become available).

Indeed, there is an undisputed role for immunoassays in surveillance studies, which may guide public health planning and help to define the trajectory of the epidemic. Their potential role for targeting vaccination in certain subgroups is being investigated. Some have suggested that immunoassays could identify previously infected and recovered healthcare workers and other essential workers who could potentially return to work, on the assumption that they are immune to reinfection. Whether this is the case, and for how long immunity lasts in the case of COVID-19, remains unclear. Therefore the validity of the concept of 'immunity passports' remains unclear. ${ }^{[40]}$

\section{HIV-COVID-19 co-infected persons}

There are currently no data on how diagnostic, management and prognostic considerations may be different in HIV-infected $v$. uninfected persons. In HIV-infected patients presenting with a respiratory tract infection, the general possibilities outlined above have to be considered, including considering Pneumocystis carinii pneumonia and $\mathrm{TB}$ in the differential diagnosis. Although well documented, it is not widely appreciated that between 10 and $20 \%$ of community-acquired pneumonia or acute lower respiratory tract infection in sub-Saharan Africa and parts of Asia is due to Mycobacterium tuberculosis ${ }^{[1-43]}$, and this proportion is even higher in HIV-infected persons. ${ }^{[1,43]}$ It is also possible that COVID-19 infection may unmask subclinical TB in both HIV-infected and uninfected persons. On the other hand, and particularly in HIV-infected persons, COVID-19 pneumonia, as in the case of influenza, ${ }^{[43]}$ may be associated with a poorer prognosis in hospitalised patients with TB. Whether the SARS-CoV-2 viral load will be higher in HIV-infected persons, and therefore RT-PCR sensitivity better, remains unclear. In HIV-uninfected persons, co-infection with more than one pathogen has already been documented, e.g. co-infection with COVID-19 and influenza and/or bacterial organisms. ${ }^{[4]}$ It is possible that co-infection with more than one pathogen may be more frequent 
in HIV-infected persons or those with advanced immunosuppression. These unanswered questions will only be resolved once more data become available.

\section{Summary and conclusions}

The rapidly spreading COVID-19 pandemic has exposed capacity weaknesses in healthcare and laboratory testing systems. Although the mainstay of testing remains RT-PCR, there are several drawbacks, including a significant false negative rate in the early course of the disease, assay cost and lack of assay simplicity and the requirement for complex laboratory infrastructure. There is an emerging shortage of reagents, including RNA extraction kits, that is likely to worsen; already many centres are using dry nasopharyngeal swabs because of the shortage of viral transport medium. Antibody-based immunoassays have been developed, although they have a limited role in the early diagnosis of symptomatic patients. Their incremental benefit over RTPCR assays, and their role in other applications, including surveillance and targeting of individuals for vaccination and redeployment into the workforce, are under investigation.

\section{Declaration. None.}

Acknowledgements. None.

Author contributions. KD conceived the idea and drafted the manuscript. All the co-authors contributed to specific sections and critically appraised the manuscript

Funding. KD, SJ, MD, JWC, AP, EM, PG and AE are supported by the SA MRC (RFA-EMU-02-2017), EDCTP (TMA-2015SF-1043, TMA1051-TESAII , TMA-CDF2015), UK Medical Research Council (MR/ S03563X/1) and the Wellcome Trust (MR/S027777/1).

Conflicts of interest. None.

Special disclosure. For maximum visibility and benefit during the COVID-19 pandemic, concurrent publication will occur in the Wits Journal of Clinical Medicine (https://journals.co.za/content/journal/wjcm) and the African Journal of Thoracic and Critical Care Medicine (http:// www.ajtccm.org.za/index.php/SARJ/index), with permission from the editors of both journals. Both are open-access journals.

1. World Health Organization. WHO Coronavirus disease (COVID-2019) situation reports, 2020. Geneva: WHO, 2020. https://www.who.int/emergencies/diseases/ novel-coronavirus-2019/situation-reports/ (accessed 28 March 2020).

2. Centers for Disease Control and Prevention. Evaluating and testing persons for coronavirus disease 2019 (COVID-19). https://www.cdc.gov/coronavirus/2019$\mathrm{nCoV} / \mathrm{hcp} /$ clinical-criteria.html (accessed 7 April 2020)

3. Infectious Diseases Society of America. COVID-19 prioritisation of diagnostic testing. https://www.idsociety.org/globalassets/idsa/public-health/covid-19-prioritisation-ofdx-testing.pdf (accessed 7 April 2020)

4. Hong KH, Lee SW, Kim TS, et al. Guidelines for laboratory diagnosis of coronavirus disease 2019 (COVID-19) in Korea. Ann Lab Med 2020;40(5):351-360.

5. National Institute for Communicable Diseases. Guidelines for case-finding, diagnosis, management and public health response in South Africa. https://www.nicd.ac.za/ diseases-a-Z-index/covid-19/covid-19-guidelines (accessed 8 April 2020).

6. Lippi G, Plebani M. Procalcitonin in patients with severe coronavirus disease 2019 (COVID-19): A meta-analysis. Clin Chim Acta 2020;505:190-191. https://doi. org $/ 10.1515 / \mathrm{cclm}-2020-0198$

7. Lippi G, Plebani M. Laboratory abnormalities in patients with COVID-2019 infection. Clin Chem Lab Med 3 March 2020;(epub ahead of print).

8. Wang W, Xu Y, Gao R, et al. Detection of SARS-CoV-2 in different types of clinical specimens. JAMA 2020;(epub ahead of print). https://doi.org/10.1001/jama.2020.3786
9. Russell TW, Hellewell J, Jarvis CI, et al. Estimating the infection and case fatality ratio for COVID-19 using age-adjusted data from the outbreak on the Diamond Princess cruise ship. Eurosurveillance 2020;25(12). https://doi.org/10.2807/1560-7917. es.2020.25.12.2000256

10. Guan WJ, Liang WH, Zhao Y, et al. Comorbidity and its impact on 1590 patients with Covid-19 in China: A Nationwide Analysis. Eur Respir J 2020;55(4). https://doi.org/10 .1183\%2F13993003.00547-2020

11. Wolfel R, Corman VM, Guggemos W, et al. Virological assessment of hospitalised patients with COVID-2019. Nature 2020;(epub ahead of print).. https://doi.org/10.1038/ s41586-020-2196-x

12. Zhou M, Zhang X, Qu J. Coronavirus disease 2019 (COVID-19): A clinical update Front Med 2020;(epub ahead of print). https://doi.org/10.1007/s11684-020-0767-8

13. Jordan RE, Adab P, Cheng KK. Covid-19: Risk factors for severe disease and death. BMJ 2020;368:m1198. https://doi.org/10.1136/bmj.m1198

14. Pan Y, Long L, Zhang D, et al. Potential false-negative nucleic acid testing results for severe acute respiratory syndrome coronavirus 2 from thermal inactivation of samples with low viral loads. Clin Chem 2020;(epub ahead of print). https://doi.org/10.1093/ clinchem/hvaa091

15. Yang Y, Yang M, Shen C, et al. Evaluating the accuracy of different respiratory specimens in the laboratory diagnosis and monitoring the viral shedding of 2019-nCoV infections. BMJ 2020;(epub ahead of print). https://doi.org/10.1101/2020.02.11.20021493

16. Wikramaratna P, Paton RS, Ghafari M, Lourenco J. Estimating false-negative detection rate of SARS-CoV-2 by RT-PCR. BMJ 2020;(epub ahead of print). https://doi. org/10.1101/2020.04.05.20053355

17. Ai T, Yang Z, Hou H, et al. Correlation of chest CT and RT-PCR testing in coronavirus disease 2019 (COVID-19) in China: A report of 1014 cases. Radiology 2020:200642; (epub ahead of print). https://doi.org/10.1148/radiol.2020200642

18. Fang Y, Zhang H, Xie J, et al. Sensitivity of Chest CT for COVID-19: Comparison to RT-PCR. Radiology 2020:200432;(epub ahead of print). https://doi.org/10.1148/ radiol.2020200432

19. Li Y, Yao L, Li J, et al. Stability issues of RT-PCR testing of SARS-CoV-2 for hospitalised patients clinically diagnosed with COVID-19. J Med Virol 2020;(epub ahead of print). https://doi.org/10.1002/jmv.25786

20. Xie X, Zhong Z, Zhao W, Zheng C, Wang F, Liu J. Chest CT for typical 2019-nCoV pneumonia: Relationship to negative RT-PCR testing. Radiology 2020:200343;(epub ahead of print). https://doi.org/10.1148/radiol.2020200343

21. Yu F, Yan L, Wang N, et al. Quantitative detection and viral load analysis of SARS-CoV-2 in infected patients. Clin Infect Dis 2020;(epub ahead of print). https://doi.org/10.1093/ $\mathrm{cid} / \mathrm{ciaa} 345$

22. World Health Organization. Coronavirus disease (COVID-19) technical guidance: Surveillance and case definitions. https://www.who.int/emergencies/diseases/novelcoronavirus-2019/technical-guidance/surveillance-and-case-definitions (accessed 7 April 2020

23. Chang D, Mo G, Yuan X, et al. Time kinetics of viral clearance and resolution of symptoms in novel coronavirus infection. Am J Respir Crit Care Med 2020;(epub ahead of print). https://doi.org/10.1164/rccm.202003-0524le

24. Zhifeng J, Feng A, Li T. Consistency analysis of COVID-19 nucleic acid tests and the changes of lung CT. J Clin Virol 2020;(epub ahead of print). https://doi.org/10.1016/j. jcv.2020.104359. 2020.

25. Wu F, Zhao S, Yu B, et al. A new coronavirus associated with human respiratory disease in China. Nature 2020;579(7798): 265-269. https://doi.org/10.1038/s41586-020-2008-3

26. Li C, Debruyne DN, Spencer J, et al. High sensitivity detection of coronavirus SARS-CoV-2 using multiplex PCR and a multiplex-PCR-based metagenomic method. bioRxiv 2020:2020.03.12.988246;(epub ahead of print). https://doi. org/10.1101/2020.03.12.988246

27. Vogels CBF, Brito AF, Wyllie AL, et al. Analytical sensitivity and efficiency comparisons of SARS-COV-2 qRT-PCR assays. medRxiv 2020:2020.03.30.20048108; (epub ahead of print). https://doi.org/10.1101/2020.03.30.20048108

28. Dong L, Zhou J, Niu C, et al. Highly accurate and sensitive diagnostic detection of SARS-CoV-2 by digital PCR. medRxiv 2020:2020.03.14.20036129; (epub ahead of print). https://doi.org/10.1101/2020.03.14.20036129

29. Kuypers J, Jerome KR. Applications of digital PCR for clinical microbiology. J Clin Microbiol 2017;55(6):1621-8. https://doi.org/ 10.1128/JCM.00211-17

30. World Health Organization. Laboratory testing for coronavirus disease (COVID-19) in suspected human cases. Interim Guidance, 19 March 2020. https://apps.who.int/iris/ handle/10665/331501 (accessed 8 April 2020).

31. Foundation for Innovative New Diagnostics. SARS CoV-2 diagnostic pipeline. https:/ www.finddx.org/covid-19/pipeline/ (accessed 9 April 2020).

32. John Hopkins Bloomberg School of Public Health Centre for Health Security. Serology-based tests for COVID-19. http://www.centerforhealthsecurity.org/resources/ COVID-19/serology/Serology-based-tests-for-COVID-19.html\#sec2 (accessed 10 April 2020). 
33. Vashist S. In vitro diagnostic assays for COVID-19: Recent advances and emerging trends. Diagnostics 2020;10(4):202. https://doi.org/10.3390/diagnostics 10040202

34. Business Insider. Spain, Europe's worst-hit country after Italy, says coronavirus tests it bought from China are failing to detect positive cases. https://www.businessinsider.com/ coronavirus-spain-says-rapidtests-sent-from-china-missing-cases-2020-3?r=DE\&IR=T (accessed 9 April 2020).

35. To KK, Tsang OT, Leung WS, et al. Temporal profiles of viral load in posterior oropharyngeal saliva samples and serum antibody responses during infection by SARSCoV-2: An observational cohort study. Lancet Infect Dis 2020;(epub ahead of print). https://doi.org/10.1016/S1473-3099(20)30196-1

36. Zhao J, Yuan Q, Wang H, et al. Antibody responses to SARS-CoV-2 in patients of novel coronavirus disease 2019. medRxiv 2020:2020.03.02.20030189; (epub ahead of print). https://doi.org/10.1093/cid/ciaa344

37. Guo L, Ren L, Yang S, et al. Profiling early humoral response to diagnose novel coronavirus disease (COVID-19). Clin Infect Dis 2020; (epub ahead of print). https:// doi.org/10.1093/cid/ciaa310

38. Okba NMA, Muller MA, Li W, et al. Severe acute respiratory syndrome coronavirus 2 -specific antibody responses in coronavirus disease 2019 patients. Emerg Infect Dis 2020;26(7);(epub ahead of print). https://doi.org/10.3201/eid2607.200841

39. Hsueh PR, Huang LM, Chen PJ, Kao CL, Yang PC. Chronological evolution of $\operatorname{IgM}, \operatorname{IgA}, \operatorname{IgG}$ and neutralisation antibodies after infection with SARS-associated coronavirus. Clin Microbiol Infect 2004;10(12):1062-1066. https://doi.org/10.1111/ j.1469-0691.2004.01009.x
40. Kuldeep D, Khan S, Ruchi T, et al. COVID-19, an emerging coronavirus infection: advances and prospects in designing and developing vaccines, immunotherapeutics, and therapeutics. Human Vaccines \& Immunotherapeutics 2020; (epub ahead of print). https://doi.org/10.1080/21645515.2020.1735227

41. Nyamande K, Lalloo UG, John M. TB presenting as community-acquired pneumonia in a setting of high TB incidence and high HIV prevalence. Int J Tuberc Lung Dis 2007;11(12):1308-1313.

42. Vray M, Germani Y, Chan S, et al. Clinical features and aetiology of pneumonia in acidfast bacillus sputum smear-negative HIV-infected patients hospitalised in Asia and Africa. AIDS 2008;22(11):1323-1332. https://doi.org/10.1097/qad.0b013e3282fdf8bf

43. Walaza S, Tempia S, Dawood $\mathrm{H}$, et al. Influenza virus infection is associated with increased risk of death amongst patients hospitalised with confirmed pulmonary tuberculosis in South Africa, 2010 - 2011. BMC Infect Dis 2015;15:26. https://doi. org/10.1186/s12879-015-0746-x

44. Khodamoradi Z, Moghadami M, Lotfi M. Co-infection of coronavirus disease 2019 and influenza: A report from Iran. Preprints 2020,2020030291;(epub ahead of print). https://doi.org/10.34172/aim.2020.04

Accepted 16 April 2020. 